\title{
Ejemplo de trabajo de desarrollo y cooperación en contextos interculturales Perfil: Sofía Pinzón Millán en World Refugee*
}

\author{
Ximena Amado**
}

Recibido: 30 de agosto de 2013

Evaluado: 21 de septiembre de 2013

Aceptado: 4 de octubre de 2013

\section{Resumen}

Colombia recibe cooperación internacional dada sus circunstancias políticas, climáticas y económicas, porque el país no tiene aún un nivel alto de educación, salud y de esperanza de vida. Sin embargo, hace pocos años, Colombia pasó de ser un país subdesarrollado a uno en vía de desarrollo. Esta experiencia permite que hoy hayan más profesionales jóvenes colombianos interesados en aportar sus conocimientos en las zonas más necesitadas del país. No obstante, es inusual encontrar a un colombiano lejos de casa realizando proyectos de desarrollo. $\mathrm{Al}$ respecto, vale la pena preguntarse si el perfil colombiano permite realizar el trabajo de manera más fluida o si, por el contrario, las dimensiones culturales colombianas generan malentendidos. Para conocer la respuesta, se presenta a continuación el perfil laboral de Sofía Pinzón Millán, como ejemplo de relaciones interculturales exitosas y liderazgo de colombianos en el exterior en proyectos de desarrollo y sus posibles ventajas interculturales.

Palabras clave: interculturalidad, cooperación, desarrollo, ONG, organizaciones internacionales.

* Reporte de caso. Este trabajo se ha realizado de manera individual durante la cátedra Cooperación para el Desarrollo y Organizaciones Internacionales, dictada por el doctor Bernhard Zimmermann, profesor experto en sociología. Su experiencia laboral se destaca por sus trabajos en desarrollo para la Organización Internacional de Trabajo, la fundación Friedrich Ebert y la Sociedad Alemana para el Trabajo Conjunto Internacional en África del oeste. Sus temas de enfoque han sido las relaciones interétnicas, el desarrollo de ciudades, las investigaciones, la docencia y la cooperación intercultural en el desarrollo. En esta clase se profundiza en el campo laboral del desarrollo y la cooperación internacional. Para tener un punto de vista real, se realizan varias entrevistas a personas que se desenvuelvan en este campo en el ámbito mundial. Esta clase hace parte del área de Desarrollo Laboral de la Maestría de Comunicación Intercultural y Cooperación de la Universidad de Ciencias Aplicadas de Múnich (http://www. gs.hm.edu/interkulturelle_kommunikation_kooperation/master/index.de.html).

** Estudiante de quinto semestre de la Maestría en Comunicación Intercultural y Cooperación de la Universidad de Ciencias Aplicadas de Múnich. 


\section{Example for the development and cooperation work in intercultural context}

\section{Profile: Sofía Pinzón in World Refugee}

\begin{abstract}
Under the context of developing work in countries of the globalized world, Colombia is known for being a country that accepts international aid. The Colombian experience with this type of cooperation is to adopt and support projects, in the best cases, learn to sustain them independently. This inspires many young people who hope to contribute to the national development. However, it is unusual to find a Colombian far away from home carrying out development projects, even more exceptional, executing international cooperation work in Asia Pacific.
\end{abstract}

In this regard, it is worth asking whether the profile Colombian profile allows working more smoothly or if, on the contrary, the Colombian cultural dimensions generate more than usual misunderstandings and difficulties. In order to provide a positive account, it is presented below the profile of Sofia Pinzón Millán as an example of successful intercultural relations and leadershipof Colombians in the field of development projects. Within the description of the profile of Sofia Pinzón, it will relate concepts related to intercultural communication, team work within international development and cooperation, and cultural dimensions. The exposure of this profile was conducted by interviewing Sofía Pinzón and in the context of the class of Development Cooperation and International Organizations of the Masters in Intercultural Communication and Cooperation in the Munich University of Applied Sciences (MUAS).

Keywords: interculturalism, cooperation, development, NGOs, international organizations.
Received: August 30, 2013

Evaluated: September 21, 2013

Accepted: October 4, 2013 


\title{
Exemplo de trabalho de desenvolvimento e cooperação em contextos interculturais Perfil: Sofía Pinzón Millán en World Refugee
}

\begin{abstract}
Resumo
Recebido: 30 de agosto de 2013

No contexto dos países que precisam aceitar a ajuda estrangeira e cooperação internacional, e dadas às circunstâncias políticas, climáticas ou econômicas, entre outros, encontra-se a Colômbia. As ajudas são aceitas porque o país não tem um alto nível de educação, saúde e expectativa de vida, e ainda não tem capacidade de resolver seus próprios problemas.

No entanto, há alguns anos atrás, a Colômbia deixou de ser um país subdesenvolvido, para ser um em desenvolvimento ou de renda média, o que implica que diminui a ajuda internacional então vai para um país que precisa mais dela. A experiência colombiana, com este tipo de cooperação é adotar projetos de apoio, e na melhor das hipóteses, aprender a sustentar de forma independente. Isto inspira a muitos jovens que esperam contribuir para o desenvolvimento do país, e cada vez há mais profissionais jovens interessados em fornecer seus conhecimentos nas regiões mais carentes da Colômbia. No entanto, é raro encontrar um colombiano longe da sua casa levando a cabo projetos de desenvolvimento ou, ainda mais excepcional, executando trabalhos de cooperação internacional na Ásia-Pacífico. A este respeito, vale a pena perguntar se o perfil colombiano permite realizar o trabalho de forma mais suave, ou se, pelo contrário, as dimensões culturais colombianas geram desentendimentos ou dificuldades.
\end{abstract}


Para conhecer a resposta, é apresentado a seguir o perfil Sofia Pinzón Millán, como um exemplo de relações interculturais de sucesso e liderança de colombianos no exterior em projetos de desenvolvimento. Na descrição do perfil da Sofia Pinzón, relacionar-se-á conceitos relacionados com a comunicação intercultural, trabalhos em equipes internacionais, desenvolvimento e de cooperação, bem como algumas dimensões culturais.

A exposição deste perfil foi realizada com uma entrevista a Sofia Pinzón e no contexto da classe de Cooperação para o Desenvolvimento e Organizações Internacionais do Mestrado de Comunicação Intercultural e Cooperação da Universidade de Ciências Aplicadas de Múnich.

Palavras-chave: Interculturalidade, cooperação, desenvolvimento, ONG, organizações internacionais. 


\section{INTRODUCCIÓN}

El trabajo de cooperación internacional es un reto social, porque más allá de las grandes ayudas que los países se puedan dar unos a otros, los diferentes intereses y la sostenibilidad de los proyectos dependen de varios aspectos divergentes, a veces ajenos a la realidad y a las necesidades de aquellos que reciben la ayuda. Sin embargo, a pesar de las dificultades, por medio de una intervención intercultural real se pueden lograr grandes desarrollos, que pueden beneficiar a todas las partes involucradas.

En los proyectos de desarrollo, las organizaciones y las personas tienen objetivos muy diferentes. A su vez, las diferencias culturales que usualmente se presentan tienenun rol muy importante.En el caso de Sofía, se ha realizado la entrevista con el propósito de conocer cómo vive ella su ambiente laboral intercultural en Asia Pacífico. La labor de Sofía es la de asistente del director ejecutivo de la ong World Refugee. La ONG se ubica en Singapur, pero los trabajos de desarrollo se realizan en los países vecinos, por lo que Sofía debe comunicarse con personas de diferentes regiones, con distintos trasfondos culturales y bajo condiciones especiales. Por medio de sus historias y reflexiones, se podrán conocer los desafíos y dinámicas de este contexto laboral específico.
Figura 1. Cooperación internacional en Los Baños, Filipinas (2013)

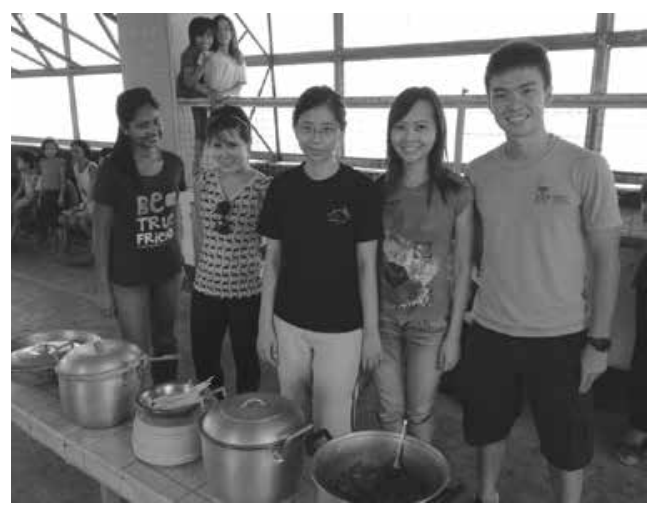

\section{LA ONG: WORLD Refugee}

World Refugee es una organización no gubernamental creada en 2012, que se enfoca en aquellas personas que han sufrido una calamidad de carácter natural, conflicto armado o cambio climático, específicamente en aquellas comunidades a las que las grandes oNg no alcanzan a llegar. A su vez, esta organización está comprometida con la construcción de la paz y con el trabajo ambiental; con ello contribuyen a la protección de personas obligadas a abandonar sus hogares en busca de refugios, debido a algunos de los anteriores factores (World Refugee, 2013). Su misión es reunir personas competentes para servir a las necesidades humanitarias de los grupos que requieren ayuda. Los expertos y voluntarios de World Refugee se encargan de proveer a pequeños y aislados grupos afectados con comida, agua, saneamiento, protección, educación y cuidado médico físico, mental y social.

El grupo principal está conformado por expertos del gremio de la cooperación internacional, como profesores y profesionales en el campo con varios años de experiencia 
en reconocidas organizaciones. El director ejecutivo es Jonathan How, quien ha tenido vasta experiencia en organizaciones internacionales como Support Mercy Relief, World Vision, wwF, Jane Goodall Institute, y hace parte del Asia Humanitarian Forum. How es la persona encargada de los proyectos, y de manera personal hace los arreglos para la asistencia y las relaciones en la ayuda desde Singapur hasta las áreas cercanas que requieren apoyo en la región Asia Pacífico.

\section{UNA COLOMBIANA EN SINGAPUR}

Sofía Pinzón Millán nació en Sabana de Torres, Santander, Colombia, hace 29 años. Realizó sus estudios de pregrado en Relaciones Internacionales y Ciencia Política y Gobierno. Durante sus estudios, viajó a Francia para perfeccionar su francés y recorrer Europa. En este viaje vivió y disfrutó de sus primeras experiencias interculturales. Su siguiente paso fue, entonces, realizar sus prácticas en Marruecos. En ese entonces su pasión se inclinaba hacia la cultura árabe, sus creencias y costumbres. Estando en Marruecos, se acercó a las comunidades islámicas, las cuales fueron inspiración para los temas de investigación de sus tesis.

Después de ampliar sus experiencias laborales, por motivos personales se presentó la oportunidad de vivir temporalmente en Singapur. La decisión fue rápidamente aceptada. Sofía es una persona que no teme a las diferencias culturales; todo lo contrario: le causan gran fascinación, y su simpatía rompe el hielo donde se presente. Pero ella sabe que eso no es suficiente; por eso, lo primero que hizo al llegar a Singapur fue empezar a conocer la región, sus dinámicas y tendencias, y, claro, seguir su recorrido profesional. En esa búsqueda, Sofía empieza a tomar cursos de Ayuda Humanitaria, Derecho Internacional Humanitario y Respuesta a Emergencia de Desastres Naturales, entre otros. A su vez, comienza a adquirir experiencia en esas áreas con la Cruz Roja. Y los entrenamientos que realizó con esta organización, aunque complicados, la capacitaron para sus siguientes pasos.

Para Sofía, estos años se resumen en un constante aprendizaje y diversión. Sus días corrieron entre el descubrimiento de nuevos territorios y el contacto con culturas muy diferentes a la propia, lo que hizo que aumentara su sentido de pertenencia con la gente y el amor por la naturaleza. La ubicación geográfica de Singapur le ha permitido a Sofía recorrer gran parte de Asia Pacífico, donde no solo ella se maravilla de todas las novedades, sino que también las personas con la que se interrelaciona se sorprenden al ver a una colombiana; de hecho, para muchos, Sofía es la primera latina que ven. La miran en la calle porque ella luce diferente. A Sofía no le molesta, porque esto le da la oportunidad de intercambiar palabras y experiencias con alguien nuevo, a la vez de encontrar algo en común en una sociedad al otro lado de su tierra natal: la apertura; ellos también son abiertos y curiosos.

\section{Sofía en World Refugee}

Sofía conoció al señor Jonathan How en un parque cuando hacía deporte en Singapur. Ella conocía la ONG, y al conversar con el señor How se sintió de acuerdo con las 
metas y la forma de trabajo de World Refugee, y le gustó el trabajo que How estaba desarrollando. Él estaba buscando apoyo, alguien con la preparación suficiente para apoyarlo. Sofía sabía que podría ser esa persona, que podría adquirir más conocimientos relacionados, y desde hace un año lo apoya, es su mano derecha.

Sofía maneja la logística, el cronograma y parte de la investigación. Ella organiza eventos para ampliar la red social y los aportes económicos. Además, se actualiza constantemente sobre las políticas y desarrollos de Asia Pacífico. Aunque ella quisiera involucrarse con grandes grupos de refugiados, el hacer parte de una ong relativamente joven y pequeña le permite realizar un trabajo más personalizado y directo; de esta manera, se evitan burocracias y conflictos en el interior del equipo. Al respecto ella cuenta:

Los países que suelen realizar cooperaciones con Maynmar, Tailandia, Filipinas, ellos tienen que cumplir con ciertos requisitos. Es por eso que cuando se escuchan noticias de esas ayudas, siempre se nombran las grandes organizaciones no gubernamentales. En ese sentido, nuestra desventaja radica en no tener todos esos contactos políticos y en no poder ayudar a miles de personas. Otra desventaja es que en Singapur no existe la cultura de la donación, entonces desarrollamosactividades para ampliarel mensaje. Hay que conocer y entender su punto de vista: ellos han generado una economía fuerte como resultado de trabajo duro; todavía siguen trabajando duro, entonces ellos no consideran que alguien deba adquirir algo gratuitamente. Dentro de las ventajas está que el dinero de Singapur se multiplica en otras regiones, como, por ejemplo, en Filipinas. Además, las relaciones son directas, la calidad de la ayuda es mejor, tú puedes ir a ver quién es quién, y quién está haciendo qué, quién necesita un tipo de ayuda y quién la otra, qué enfermedades hay. Damos una atención más personalizada a grupos aislados.

Cuando se hace trabajo de desarrollo, uno de los aspectos de mayor cuidado es la forma en que se manejan experiencias negativas, ya que de esto depende la confianza y las futuras ayudas, por ejemplo, al culpar a los socios locales o a las diferencias culturales. La confianza es una meta de gran valor, ya que son las organizaciones externas las que controlan el acceso a los recursos y, de alguna forma, tienen un poder sobre los empleados y socios. Si esto se maneja honradamente, es un manejo positivo, porque de esta forma se evitan las jerarquías nacionales y locales, las estructuras de poder y sus abusos locales, y se mantienen a la raya de conflictos que no atañen el proyecto de desarrollo.

Hüsken (2010, p. 193) dice que en la percepción de la situación llama la atención la autoevaluación positiva de los conocimientos acerca de la política, la economía y la cultura de los países que reciben la ayuda. Estas sociedades valoran el conocimiento de los expertos nativos, principalmente por los informes y el intercambio con los colegas. No solo el conocimiento profesional y el manejo del idioma es importante, sino también la aculturación, la inmersión en los procesos de información y en la vida diaria 
de las comunidades, el hacer parte del milieu (entorno físico y social inmediato en el que la gente vive o en el que algo ocurre o se desarrolla. Incluye la cultura que el individuo fue educado o vive, y las personas y las instituciones con las que interactúan, así como los estratos sociales y las jerarquías).

Esta es la recomendación real de los expertos extranjeros que han logrado desarrollar proyectos exitosos a través de experiencias positivas de cooperación. Aquellos que se integran-los "expertos híbridos" - logran tener una mejor comprensión de los aspectos que influyen en los grupos que dirigen. Sin embargo, en muchos casos, como cuenta Hüske (2010, pp. 194-208), sus relaciones se desarrollan en el milieu de las clases altas, las cuales les ofrecen informaciones académicas más amplias, pero generan escepticismo en las comunidades porque reducen la identificación que estas podrían tener con los proyectos.

En el caso de Sofía, que se desenvuelve en el área de la ayuda humanitaria, los miliues son diferentes, y ella aporta heterogeneidad al grupo de trabajo. Así, su manera de intervenir en las comunidades no se reduce a relacionarse con los grupos de millonarios que pueden apoyar la ONG, sino que hay intervención personal con los grupos afectados y el equipo intercultural de trabajo. Y este trabajo, que no solo desarrolla en la economía sino también en las posibles participaciones sociales y culturales de manera personal, hace que Sofía reafirme su ánimo en sus labores diarias. Desde el punto de vista de las dimensiones culturales, es posible que el carácter mestizo permita que el mezclarse con otros sea una actitud inconsciente y que no se sienta una diferencia con el otro que genere distancia.

\section{La Motivación de Sofía}

Desde que Sofía empezó sus carreras, su ideal era ayudar en el desarrollo y la cooperación, aunque inicialmente pensó que su trabajo transcurriría en Colombia. Cuando vivió en Europa, pudo ver de cerca cómo las organizaciones governamentales ayudan y todo el posicionamiento social que tienen; así supo que ella quería estar involucrada. Sus estudios en ciencias políticas la motivaron a crear proyectos y a revisar los aspectos políticos y la parte local; las relaciones internacionales le permitieron saber cómo conectarse con las organizaciones internacionales; y los cursos de sostenibilidad le ayudaron a saber cómo se desarrollan esos proyectos en la práctica, paso a paso y, en especial, lo más importante: cómo empezar. Ella relata que es necesario tener en cuenta cada aspecto desde el punto de vista local e internacional, que haya una aculturación y no una hegemonización en ningún sentido: ni religioso, ni político, ni geográfico, ni ecológico, ni económico, ni social, etc.

\section{SOFÍA INTERCULTURAL}

El tema de la interculturalidad está puesto sobre la mesa desde que la globalización comenzó a tener repercusiones culturales públicas. Sin embargo, aún no se ha llegado a tener una conciencia formal de cómo nos interrelacionamos entre nosotros. Muchas personas se preparan, pero les faltan ciertos talentos; otras tienen grandes capacidades sociales, pero les falta preparación; y otras tienen talento y preparación, y usan sus conocimientos y talentos para fortalecer sus debilidades y manejar de la mejor manera sus fortalezas. Sofía está en el tercer campo. 
Sabe que el ser nueva y tener un pequeño equipo reduce la posibilidad de conflictos y malos entendidos, pero está preparada para crear estrategias en caso de que estos aparezcan. Ello se nota en sus conocimientos y actitud. A continuación se presentan algunos puntos sobre su vida laboral y sus experiencias, de tal manera que haya un breve acercamiento al desarrollo de la vida de Sofía en Singapur.

\section{Sobre la ayuda a comunidades}

"Tratamos de ser familiares, somos flexibles, porque queremos que confíen en nosotros". "Trabajar con gente de diferentes contextos culturales al mío es maravilloso, en realidad. Para mí siempre fue importante saltar de un sitio a otro, conocer gente de diferentes partes del mundo, y que ellos conocieran algo de mi cultura, y conocer diferentes idiomas... Es una experiencia muy especial para mí... Singapur es para mí increíble, porque es un lugar muy pequeño, pero encuentras personas de todo lado.Es maravillo.Tú nunca sabes con qué otra cultura te vas a encontrar en la esquina, porque además viene gente de todo el mundo a conocer o a hacer negocios, y ahora con World Refugee estoy conociendo gente de Myanmar, Filipinas, Tailandia, Indonesia, y ellos se alegran de conocer gente de Colombia... Siempre es una aventura salir a la calle. Pero lo es aún más con el trabajo de cooperación, porque están involucradas muchas personas de todo el mundo: de Corea, Japón, Malasia, India...".

\section{Diferentes comportamientos}

En Singapur todavía no hay una mentalidad de que ayudar seas parte de la vida, especialmente si tienes dinero y recursos; así que ahora ellos viven una nueva y grande economía, donde se concentran en trabajar y seguir haciendo dinero y no se dan cuenta de que el siguiente paso es apoyar a otros. Pero esta oNG sabe que puede darles a entender, paso a paso, la importancia de solidarizarse y cooperar. World Refugee ha empezado a compartir su mensaje en eventos sociales, a hacerse conocer por medio de publicidad y eventos artísticos y deportivos en Singapur. Para Sofía es muy claro que no se puede juzgar ni ver de mala forma estos diferentes modos de comprender la cooperación. Las personas de Singapur no han tenido la experiencia, y cuando se les explica que dar algo gratis de corazón, hacer un regalo para ayudar, apoyar a los necesitados o, por ejemplo, asignarles trabajo no es solo dar algo gratis, entonces ellos entienden que es un aporte hacia futuro y que ellos también se verán recompensados.

\section{Mejores momentos}

"La gente, cuando tú vas a ellos, ellos saben que tú vas a ayudarles. Si tú vas donde el alcalde, seguramente las ayudas se desaparecen. Entonces, cuando tú vas a ellos directamente, al colegio, a los profesores, ellos desde que llegas están agradecidos. Y tú ves cómo los profesores trabajan, se organizan, hacen comida para trescientas personas incluso el fin de semana, y cuidan de todo".

“Ellos lo hacen porque saben que así los niños van a volver al colegio y que la comunidad va a estar organizada. Con la ayuda directa no hay burocracia,y siempre tienes el apoyo local, que es de gran ayuda, por ejemplo, para explicar y traducir. Sus opiniones nos son de gran importancia, porque realmente nos permiten cubrir sus necesidades urgentes". 
“Como colombiana, siempre estás de buen humor, sonríes, eres abierta a la gente, y eso es de gran ayuda, da confianza. Nosotros hacemos favores sin esperar nada a cambio, y yo soy muy comprometida con los favores que hago; yo me involucro y demuestro que quiero pertenecer a la comunidad".

“Mi experiencia más gratificante que ahora llega a mi mente pasó en Filipinas. Hace palpitar mi corazón, no lo puedo comparar con nada. Ellos sabes que tú vienes y que les estás llevando algo. Ves la luz en sus ojos. Tú sabes que ellos están pensando 'ellos no van a lastimarme'. Realmente pasa que hay personas que no tienen nada, absolutamente nada. Yo supe cuánto aprecian esto. Pero cuando fui a Filipinas con el programa de protección, vi a una niña que me miraba constantemente, y ella era muy tímida. Yo pienso que tendría entre trece y catorce años. Y ella mandó a su padre a hablarme, porque ella me había mandado una caja pequeña. Yo no sabía si aceptarla o no, yo no sabía qué hacer. Yo pensé que iba a saltar un sapito de la caja, no sé [risas]. Entonces abrí la cajita y había un pedacito de pastel que ella me regalaba. Ella estaba tan agradecida. Yo no sabía qué hacer, solo trataba de no llorar, ¡yo nunca me lo esperé! Yo solo pensaba: ‘Soy yo quien viene a traer la ayuda, a traerle algo, y ella decidió ir a comprar este pastel local y dármelo!'. Fue un gesto muy tierno por parte de ella. Ella quería mostrarme qué feliz estaban por nuestro trabajo. Y eso es lo verdaderamente gratificante. Mucha gente dona mucho dinero, pero no visitan los campos; claro que eso debe hacerte feliz, estás ayudando mucho,pero cuando vas a los campos a llevar la ayuda por ti mismo, puedes ver la carita de la gente, sus sonrisas. Es maravilloso [...]".

\section{Figura 2. Cooperación internacional} en Los Baños, Filipinas (2013)

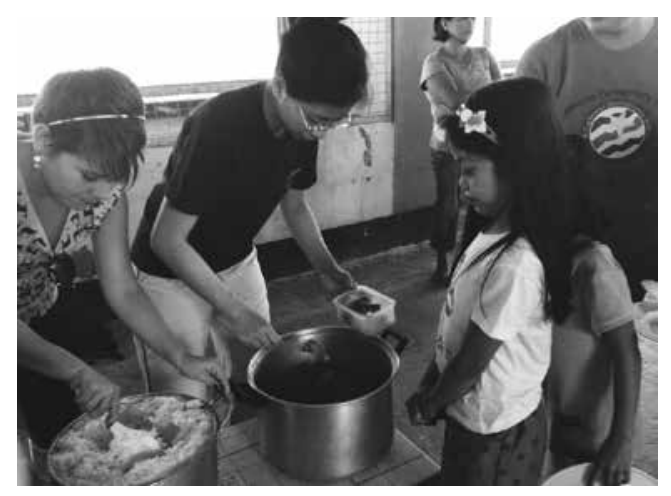

\section{Conclusiones}

\section{Every kind of peaceful cooperation among men is primarily based on mutual trust and only secondarily on institutions [...]}

Albert Einstein

La entrevista a Sofía deja una sensación muy positiva con respecto al trabajo de desarrollo. La cooperación internacional y humanitaria es una parte del trabajo social que rompe los paradigmas sobre los posibles conflictos interculturales en las sociedades actuales. Más allá de las distancias y las diferencias culturales en los distintos tipos de intercambio, hay acciones y valores culturales que nos unen, nos complementan y nos hacen más fuertes. Esas acciones pueden superar conflictos, porque las prioridades se mantienen como primordiales. El bienestar de todos y la protección es lo que define la cooperación.

Hay varios recursos que pueden ser revisados en cada situación, como, por ejemplo, determinar cuáles son los medios de comunicación más efectivos entre los grupos de 
trabajo, predecir comportamientos y prevenir y resolver conflictos. Las entrevistas actuales a diferentes líderes del trabajo de desarrollo internacional afirman que, en su mayoría, la gente que es activa en el trabajo de campo prefiere comunicarse directa y personalmente con los equipos de trabajo, cara a cara, para evitar malos entendidos. En el caso de Sofía, "textiar" (correos electrónicos y mensajes de texto) es el mejor método y el más rápido. No obstante, ella afirma que en cada caso y zona, las formas de comunicación deben ser evaluadas. Pero sin duda, la comunicación cara a cara ofrece confianza, que es una valor prodigioso en cualquier cultura y para cualquier persona.

\section{ReFERENCIAS}

Amado, X. (2013, 6 de junio). Entrevista a Sofía Pinzón Millán. Comunicación personal.

Barton, E. (2013, julio). When texting goes corporate, skip the "LOL". BBC, Capital. Recuperado dehttp://www.bbc.com/capital/story/ 20130521-lol-boss-and-other-faux-pas/1

Hüsken, T. (2006). Der Stamm der Experten.Bielefeld:TranscriptVerlag.

Hüsken, T. (2010). Outside the whale: the contested life and work of development experts. Journal fürEntwicklungspolitik, 26(3).

Pfeiffer, C. (2007).Die Erfolgskontrolle der Entwicklungsarbeit und ihre Realitäten.Bielefeld:Transcript Verlag.

World Refugee(2013).www.facebook.com/World Refugee

World Refugee (2013). www.worldrefugee.org

Zimmermann, B. (2011).Interkulturelle Kooperation in der EZ:wie verhalten sich die Akteure und wie nehmen sie die Situation wahr?Recuperado de: http://w3-mediapool. hm.edu/mediapool/media/fk13/fk13_lokal/ pdfaw/9internationalertag/vortrag_bernhardt_zimmermann.pdf 